\title{
NEW EVIDENCE REGARDING THE INTERPRETATION OF CRITICAL POTENTIALS IN HYDROGEN.
}

\author{
By P. S. Olmstead.
}

SyNOPSIS.

\begin{abstract}
Critical Potentials in Hydrogen.-(I) Experimental separation of effects due to atomic and molecular hydrogen. In an ionization tube of the Lenard type, a close grid of tungsten wires was introduced between the filament and the gauze in front of the plate. When this grid was heated electrically to incandescence the proportion of atoms was greatly increased by dissociation of the molecules. (2) Effects due to radiation were distinguished from effects of ionization by comparing the electron currents to a disk electrode with those to a wire which because of its small area was relatively insensitive to effects of radiation. (3) Six critical potentials between ro and I6 volts were observed and measured with reference to the strongest break, assumed to occur at 16.0 volts. Each break point was located at the intersection of two tangents; hence the relative values are probably accurate to 2 or 3 per cent. Purified electrolytic hydrogen was used. (4) Experimental interpretation of the breaks, which agrees in general with the previous theoretical interpretation based on quantum theory, is as follows: radiation from the atom at I0.x and at 12.2 volts, corresponding to the first two terms of the Lyman series; ionization of the molecule at Ir.5 volts and of the atom at 13.6 volts; dissociation of the molecule and radiation at 12.9 volts; finally, dissociation of the molecule and ionization of one of its parts at 16.0 volts. The energy required for dissociation of the molecule is therefore equivalent to about 2.8 volts.
\end{abstract}

\section{INTRODUCTORY.}

THE conclusions, which have been published by recent investigators on the critical potentials of hydrogen, were collected and tabulated in a recent paper by Professor K. T. Compton and the author. ${ }^{1}$ Since that time, reports by Mohler, Foote, and Kurth ${ }^{2}$ and O. S. Duffendack ${ }^{3}$ and a paper by P. E. Boucher ${ }^{4}$ have been added to the list of references. The effects which have been observed fall under five heads, which are noted in Table I.

TABLE I.

\begin{tabular}{|c|c|c|}
\hline Type of Effect. & Due to & First Observed at \\
\hline Radiation....... & Atom & 10.0 to 11.0 volts \\
\hline Ionization . . . . . . . . . . . & Molecule & 10.8 to 11.5 volts \\
\hline Dissociation plus radiation..... & Molecule & 12.5 to 13.9 volts \\
\hline Ionization ..... . & Atom & 13.1 to 14.4 volts \\
\hline Dissociation plus ionization .......... & Molecule & 15.8 to 17.1 volts \\
\hline
\end{tabular}

${ }^{1}$ K. T. Compton and P. S. Olmstead, Phys. Rev., I7, 45, I92I.

${ }^{2}$ Mohler, Foote and Kurth, PHys. Rev., I9, 4I4, I922.

${ }^{3}$ O. S. Duffendack, Phys. Rev., I9, 533, I922.

${ }^{4}$ P. E. Boucher. Phys. Rev., I9, I89, I922. 
Except in the work of Mohler, Foote, and Kurth and of Duffendack, the interpretations which appear in the second column of the table are the result of comparison of observed radiation and ionization potentials with the theoretical potentials based on some theory of the hydrogen molecule. The present paper deals with an experimental separation of the effects which are due to the atom from those which are due to the molecule.

\section{Apparatus. Treating of its Special Features.}

The apparatus used in this work was the one which was described in a previous paper ${ }^{1}$ as one to "permit of more refined observations to test some of the considerations involved in the problem." In Fig. I it is

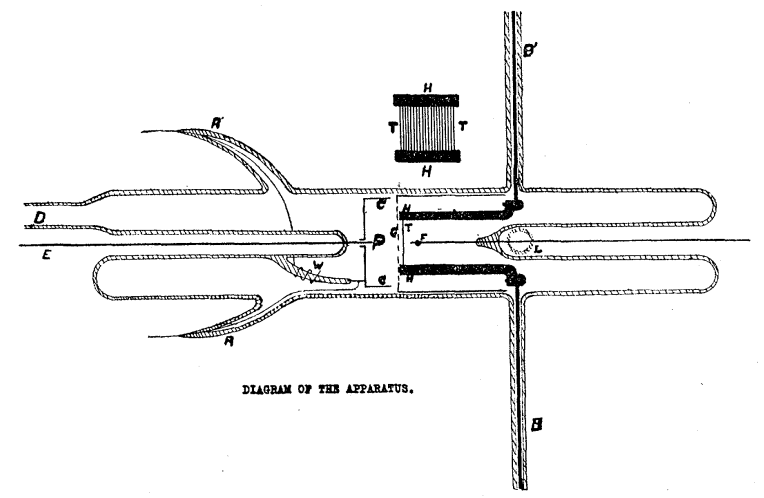

Fig. 1.

seen in cross-section. It consisted of a glass tube containing the usual elements of a Lenard typ of ionization tube, with the addition of a heating arrangement placed as close as possible to the gauze. At $F$ was a spiral tungsten filament (seen in cross-section, end on), across which the voltage drop was I.6 to I.7 volts for a current of 3.5 to 4.0 amperes. The spiral consisted of three turns only, in the middle of the filament. Since this part of the filament was hotter than the rest, and since the potential drop across the spiral part was but a small fraction of a volt, the filament was practically an equipotential source of electrons. At $G$ was a gauze covering one end of a platinum cylinder, which was connected with the galvanometer by a lead through a side-tube $L$, in order to measure the total electron emission from the filament. As close as possible to the gauze and between it and the filament, was a heating grid, consisting of twenty fine tungsten wires $T T$ strung between two copper plates $H H$ so that the wires of the grid were perpendicular to the filament.

${ }^{1}$ Loc. cit. 
The copper plates were insulated from the gauze and its supporting cylinder by small quartz blocks. The electrical connections to the grid were made by means of heavy copper wires to the plates sealed through the long glass tubes $B B^{1}$ with De Khotinsky cement at their ends. It was necessary to keep these tubes very cool to prevent the cement from melting due to the heat conducted along the wires. The second special feature of the apparatus was the receiving electrode or plate. This consisted of two parts - a single platinum wire $P$ of small cross-sectional area, and a platinum cup $C$ which surrounded $P$ and could either be joined to $P$ or insulated from it. When joined and connected to the electrometer the combined effect of radiation and ionization was observed, while if $P$ alone were connected to the electrometer, ionization only was measured, since the area of $P$ was too small to intercept enough radiation to give an appreciable photoelectric effect. Suitable guard rings were placed at strategic points to prevent leakage to the electrometer. One of these is shown at $W$. At $D$ was a connection to the diffusion pump, McLeod gauge, and source of hydrogen. Between them and the tube were two liquid air traps, the one nearest the tube being filled with coconut charcoal.

The hydrogen was obtained as in the previous work by the electrolysis of sulphuric acid. The processes involved in purifying the gas are explained in the previous paper. The tube was "baked out" by heating in a furnace to about $300^{\circ} \mathrm{C}$., keeping the tube pumped out at the same time. It was further cleansed of the unwelcome residual gas by "washing out" with hydrogen, the hydrogen being introduced and pumped out at various times during the baking.

The tungsten grid $T T$, placed as close to the region of effective electron impacts as possible without introducing disturbing effects from positive thermions, served to vary the proportion of dissociated, or atomic, hydrogen. ${ }^{1}$ Any effect which was relatively enhanced by raising the grid to a high temperature must be due to atomic hydrogen. Similarly, any effect which was relatively more pronounced when detected by the point $P$ than by the cup $C$ was attributed to ionization.

The method of obtaining the experimental readings was such as to give the greatest relative accuracy. Under the same conditions of gas pressure and current flow in the gas, as observed in the galvanometer deflections, it was possible to duplicate the values of the electrometer readings at specified accelerating voltages to within two or three per cent. in different runs, even in the region of greatest variation of the electrometer readings with the accelerating voltage, i.e., in the region from

${ }^{1}$ Langmuir, Jour. Amer. Chem. Soc., 37, 4I7, I9I5. 
I 5 to I 6 volts. In fact the individual curves from which the "Grid off for Radiation" average curve (Fig. 2) was obtained differed from the average by less than one per cent.

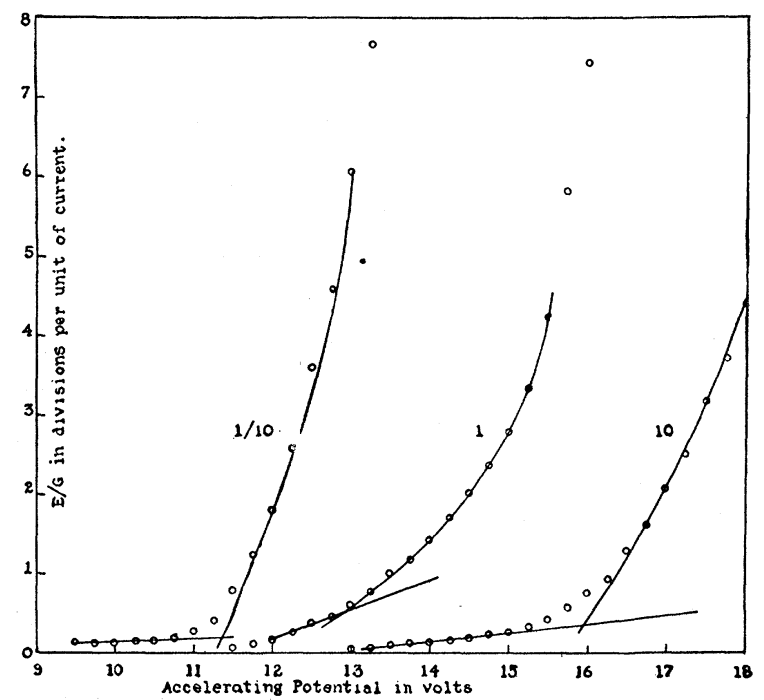

Fig. 2.

"Grid off for Radiation" Curve Breaks: $11.4 ; 12.9 ; 16.0$.

\section{ExPERIMENTAL Results.}

In this tube are two totally independent elements which may be varied independently, and hence we expect to find four types of experimental results. First, we may connect only the platinum point $P$ to the electrometer, and have the grid on. Curves obtained with this set-up are called "Grid on for Ionization" curves and will be denoted by $I$ (on). Next, we may keep the electrometer connections the same but turn the grid off. This gives a set of "Grid off for Ionization" curves and they will be denoted by $I$ (off). The two other possibilities are obtained by connecting the cup $C$ as well as the point $P$ to the electrometer, giving the arrangement suitable for observing the combined effect of radiation and ionization. We then get the sets of curves- "Grid on for Radiation," $R$ (on), and "Grid off for Radiation," $R$ (off). These four types of curves are illustrated by the average curves (Figs. 2, 3, 4 and 5), each of which is the average of a number of independent runs. These curves have been corrected from the observed relations by adjusting the accelerating voltage on each of the original curves so that the big "break," which corresponds to dissociation of the molecule plus ionization of one 
of its parts, may come at I6.o volts. The adjustment was an empirical one which made the line representing the slope of the curve after passing the break point cut the line representing the slope of the curve before reaching the break point. Since this intersection depends largely on the

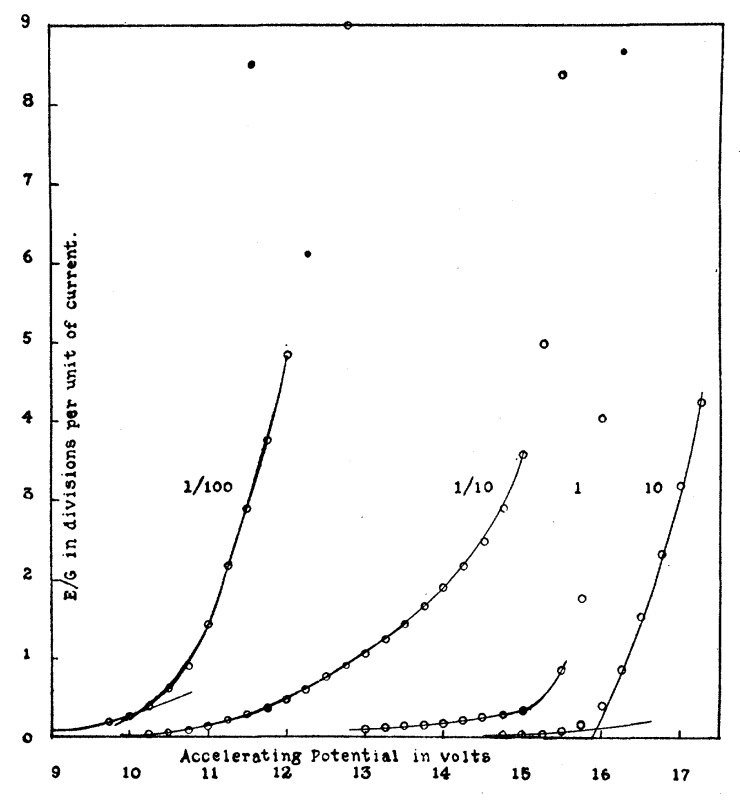

Fig. 3.

"Grid off for Ionization" Curve Breaks: $10.1 ; 11.5 ; 16.0$.

way in which the intersecting lines are chosen, the plots were consistently made so that the line on either side of the break point was fairly straight for a region of two volts. Velocity distribution curves were not generally taken, since the object of this work was to find the peculiarities of the lower breaks and to locate their positions relative to the large breaks.

In order to obtain a convenient comparison between the different effects, four ratio curves were computed. These are based on the four average curves typifying the four sets of experimental results. Each of these average curves shows the average of several experimental curves which were taken under approximately similar conditions of pressure of hydrogen, and, to a certain extent, of electron current as given by the galvanometer readings. With regard to this it was found that, if the pressure and accelerating voltage were kept constant, the ratio of the electrometer reading to the galvanometer reading $E / G$ would be approximately constant. Thus in all these results it is this ratio, or the electrometer deflection per unit bombarding current of electrons, which 


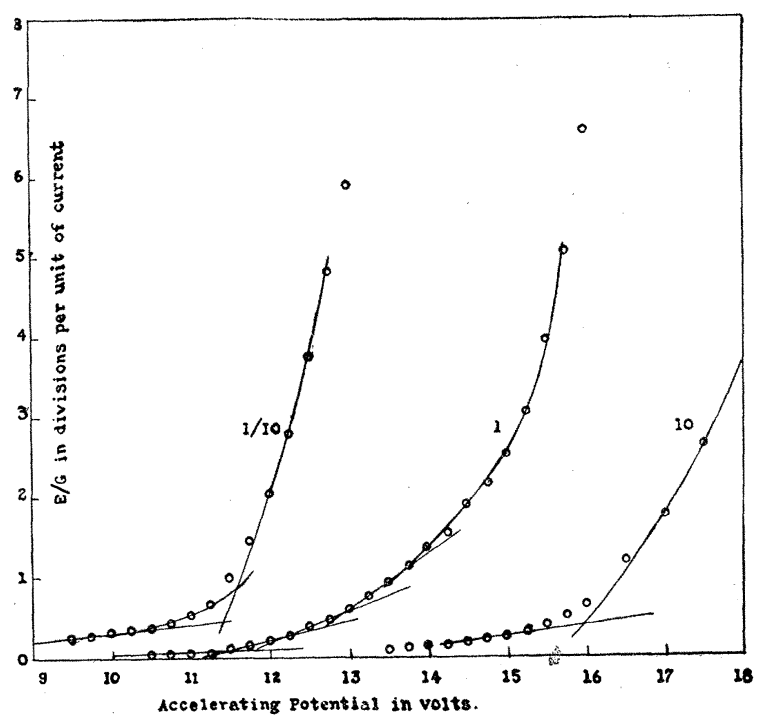

Fig. 4.

"Grid on for Radiation" Curve Breaks: $10.2 ; 11.4 ; 12.2 ; 12.9 ; 13.7 ; 16.0$.

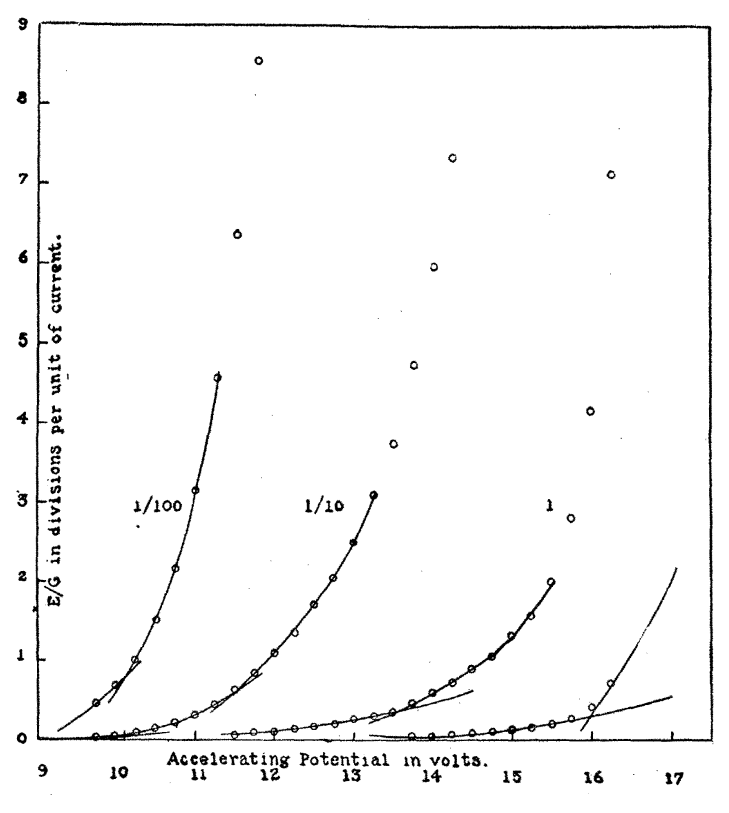

Fig. 5.

"Grid on for Ionization" Curve Breaks: $10.1 ; 11.5 ; 13.6 ; 16.0$. 
has been plotted against the accelerating voltage. Then from these curves were obtained the ratio curves:

$$
\frac{I \text { (on) },}{I \text { (off) }} \quad \frac{R \text { (on), }}{R \text { (off) }} \quad \frac{R \text { (on), }}{I \text { (on) }} \quad \frac{R \text { (off) }}{I \text { (off) }}
$$

These are shown in Figs. 6, 7, 8 and 9. The values for the points for

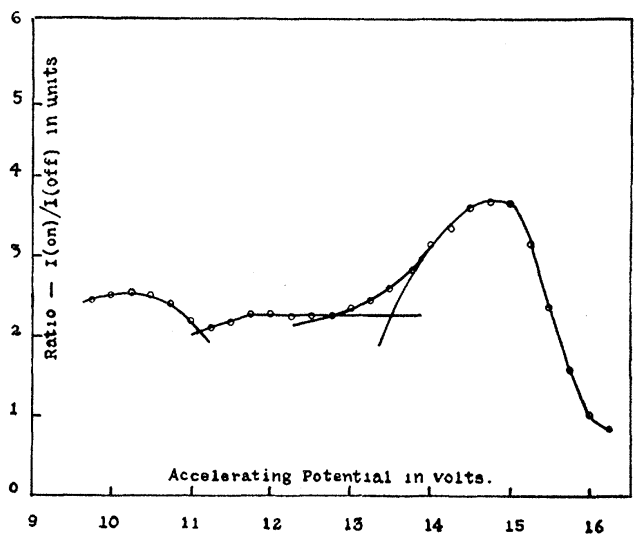

Fig. 6.

Ratio Curve for $I$ (on) $/ I$ (off).

these ratio curves were obtained from the calculated values of the averages rather than by reading the curves, so that the ratio curves themselves are experimental curves and have not been smoothed by smoothing the average curves in any way.

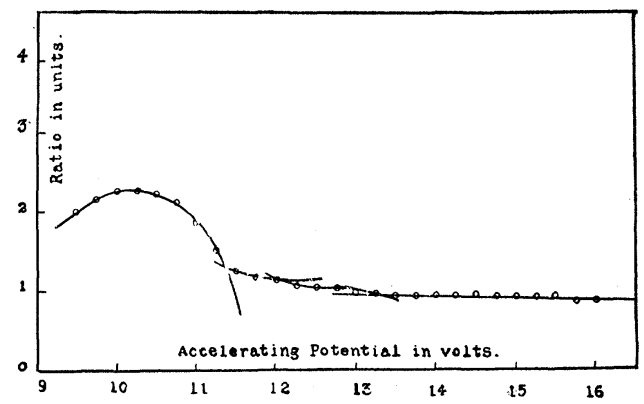

Fig. 7.

Ratio Curve for $R$ (on) $/ R$ (off).

Discussion of Results.

The interpretation of these results will be considerably facilitated if first a careful study is made of the way in which the individual ratio curves 
should behave, Take, for instance, the ratio $I$ (on)/I (off), Fig. 6 . With the grid on we should get a greater concentration of atomic hydrogen; hence when this ratio is greater than unity it means that the effect due to the atom has been increased relative to that due to the molecule. Thus, any effect which tends to bend the ratio curve toward the unity

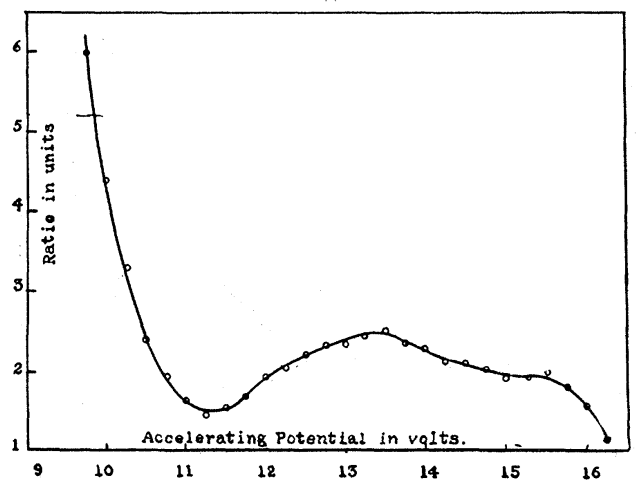

Fig. 8.

Ratio Curve for $R$ (on)/I (on).

line would be interpreted as an increase in an effect due to the molecule while anything which turns the slope away from the unity line would be taken as due to the atom. The same thing may be said of the ratio $R$ (on) $/ R$ (off), Fig. 7. Now let us consider the conclusions which may be drawn

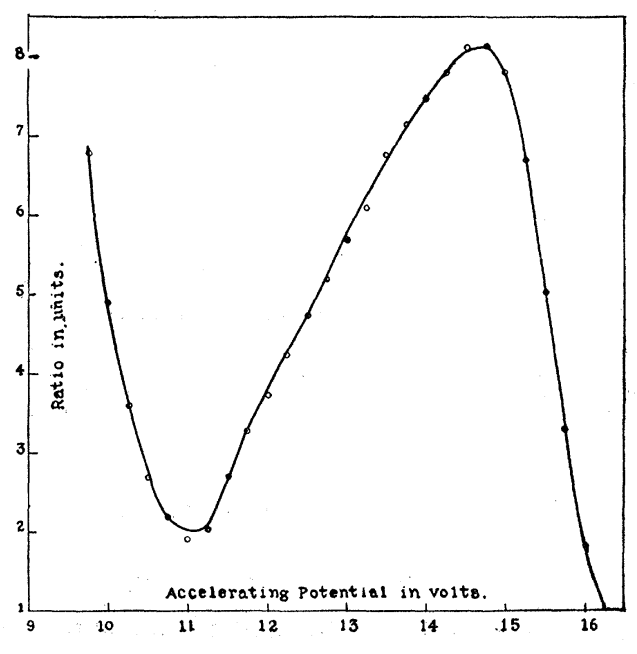

Fig. 9.

Ratio Curve for $R$ (off) $/ I$ (off) 
from the two curves. If the ionization curves should show the increased effect due to the hot grid, but the radiation curves should not, the effect itself would be taken as ionization and also as due to the atom. In like manner it is possible to get further differentiations of ionization and radiation.

TABLE II.

Data Obtained from the Ratio Curves.

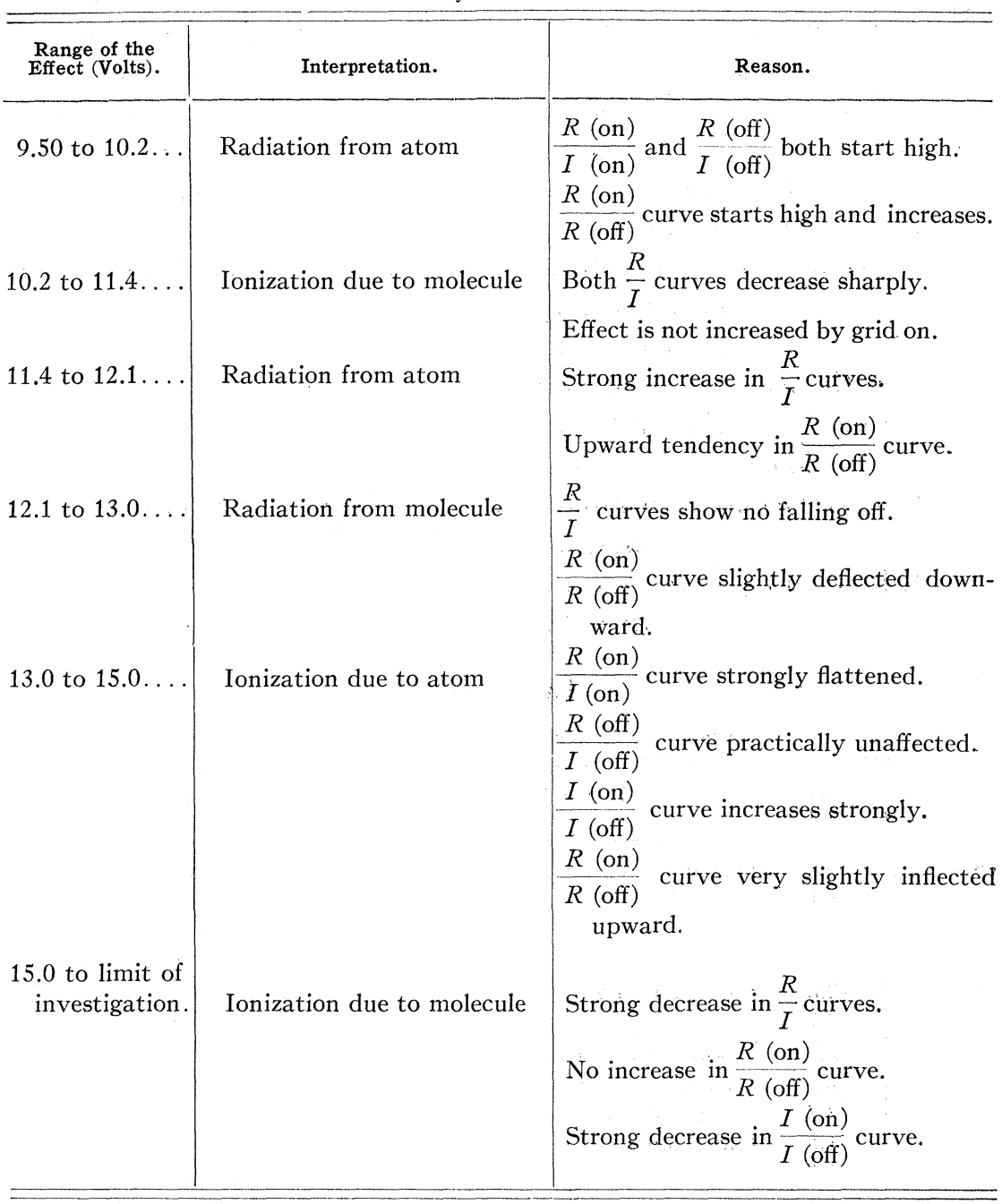

The other two ratio curves also have certain peculiarities. The grid remains unchanged while the type of effect is varied. If the radiation effect is large relative to the ionization effect, the curve will be greater than unity, whereas if the effect is mainly ionization the curve will tend 
to remain near unity. Those effects which tend to bend the curve away from the unity line are due to radiation and those which tend to bend it toward the unity line are due to ionization.

Considered with this interpretation of their behavior, these four curves make it possible to construct Table II. This shows the whole field of the investigation blocked off into units, and gives the type of effect that is most important in each unit. It remains to indicate the way in which these units are consistent with the breaks that appear on the experimental curves (Figs. 2, 3, 4 and 5). As in the case of the ratio curves we will first consider what may be expected to happen in each of these average curves. With the grid off we should not expect effects due to the atoms, but on the other hand effects due to molecules would be very sharp. Thus on the "Grid off for Ionization" curve only ionization from molecules should be observed. Similarly, on the "Grid off for Radiation" curve we should observe radiation and ionization from the molecules only. On the other hand the curves with the grid on should give both the molecular and atomic effects. The "Grid on for Radiation" curve should give all the breaks and the "Grid on for Ionization" curve all the ionization but none of the radiation effects. Accordingly, if we turn to the curves themselves, we may classify our results as shown in Table III. We note that, except for the last item, just the effects expected according to the above analysis are observed. We are therefore compelled to ask the question, "Why do we get an effect due to radiation

TABLE III.

Breaks Observed on the Experimental Curves.

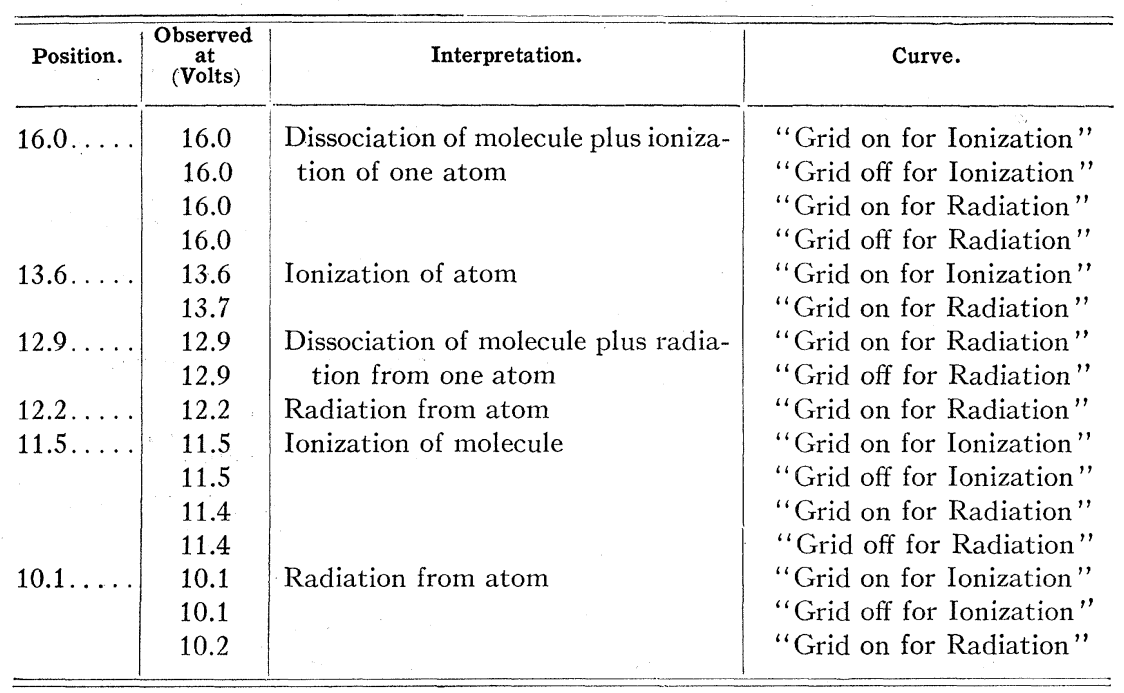


from the atom in three sets of curves when we expect it only in one?" This is easily answered when the electrical connections which were used for ionization and for radiation are considered. It is seen that in the "Grid off for Radiation" curve the effect is not observed, which is as expected. With the connections for ionization, however, there is a larger volume of gas in which the effective collisions take place and, since no ionization of any kind was detected below I I.5 volts, any small effect before that must be due to radiation. To account for a certain amount of atomic effect present even when the grid is off we turn to the filament itself. This is very hot, and besides giving off electrons, is able to decompose a certain amount of the gas near it. Whatever effect is present in the region of Io to I I volts would be more noticeable than any effect of the same order of magnitude farther up in the curve where it would be superimposed on another effect of a much larger magnitude. For this reason it is possible to detect the radiation from the IO.I volt effect even with the point $P$ alone as the detecting electrode.

In discussing these curves we should not overlook the characteristics of the breaks. We note that the break at 16.0 volts is not as sharp nor as distinct as the others but seems to start a volt or more before the steep rise in the curve takes place. We note that the other breaks are singularly distinct, so that it is usually possible to draw smooth curves on either side of the break so that they either pass through all the observed points or at most neglect one or two. The obvious explanation of this difference is that it is due to the fact that all the electrons emitted by the filament do not have the same velocity, so that it is possible for some of the electrons to have the velocity required to produce the observed effect with a smaller accelerating potential than others. For this reason we need a sliding scale to determine at what true voltage the various effects set in.

Suppose that $P_{m}$ is the probability of an electron striking a molecule of gas; $P_{a}$ the probability of striking an atom of the gas; and $p$ the probability of an electron being emitted from the filament with a velocity sufficient to produce the desired effect. $P_{m}$ will be proportional to the number of molecules in a unit volume of the gas and therefore is practically proportional to its pressure, since the partial pressure of the atoms is always small. In like manner the value of $P_{a}$ will be proportional to the number of atoms in the gas. We know that there is always a relationship between the number of atoms and molecules which remain in equilibrium at any temperature. This relationship depends on thermodynamical considerations. If we supply energy to the gas we can keep a larger proportion of the particles in the atomic form. In this work 
this was made possible by the introduction of the heating grid. Even when the grid is off a certain amount of atomic hydrogen will be in the tube due to dissociation by the filament itself. This, of course, is not formed at the place where it is most effective and thus it will be observed only when the other effects are almost negligible in comparison. To take a. numerical analogy to the situation here, suppose that there were in the case with the grid off 99 molecules to every I atom. Then the observed effects due to the molecule would be 99 times as strong as that due to the atom. Then let us suppose that with the grid on we have 90 molecules to every Io atoms. Now the effect due to the molecule is only 9 times that due to the atom, but we have also changed two other factors. The effect due to the atom has been increased io times and the effect due to the molecule has been slightly decreased, but has practically the same value as before. Thus we see we can express the probability of striking an atom in terms of the probability of striking a molecule as

$$
P_{a}=k P_{m},
$$

where $k$ is a constant depending on the linear relation of the number of atoms to the number of molecules, or in this particular case on the amount of dissociation produced by the grid. But, in addition to striking a molecule or atom, we must have a transfer of energy at the collision in order to produce the desired effect. If the electron making the collision does not have sufficient energy to produce the effect, the energy cannot be transferred. On the other hand, if the electron has more, it can, of course, give it. We know that the energy of an electron is proportional to the square of its velocity, so if we assume Maxwell's Law of Distribution of Velocities we can get an idea of the probability of having a particular velocity or more, and in so doing get a corresponding probability of having a specified amount of energy or more. This is a more difficult thing to consider. In Table IV. is given, against tenths of the mean energy, the percentage of electrons having less than the specified part of the mean energy $(F)$, the percentage of electrons having more than the specifed part of the mean energy $(\mathrm{I}-F)$, the percentage of electrons having energies in the interval between two successive specified divisions of the energies $(d F)$, and the mean energy of all electrons having energy greater than the amount specified in the first column. This is expressed in terms of the mean energy of all the electrons and in equivalent volts. Thus, to take a specific example, 50.64 per cent. of the electrons have less than 0.8 times the mean energy, 49.36 per cent. have more, and the mean energy of these 49.36 per cent. is I.6I times the mean energy of all the electrons, or 0.97 volt. The expression in equivalent volts is obtained from the 
average correction for a filament temperature of $235^{\circ}$ to $2400^{\circ} \mathrm{K}$. This has been found to be 0.60 volt. ${ }^{1}$ The values given in the last two columns were obtained by mechanical integration, the steps used being those tabulated.

TABLE IV.

\begin{tabular}{|c|c|c|c|c|c|}
\hline \multirow{2}{*}{$\begin{array}{c}\begin{array}{c}\text { No. of Times } \\
\text { Mean Energy. }\end{array} \\
\text { Times } E^{-}\end{array}$} & \multirow{2}{*}{$\begin{array}{l}F . \\
\%\end{array}$} & \multirow{2}{*}{$\frac{\mathrm{I}-F}{\%}$} & \multirow{2}{*}{$\frac{d F .}{\%}$} & \multicolumn{2}{|c|}{$\begin{array}{l}\text { Mean Energy of Grour } \\
(I-F) .\end{array}$} \\
\hline & & & & Times $\vec{E}$. & Volts. \\
\hline $0.0 \ldots \ldots \ldots$ & 0.00 & 100.00 & & 1.00 & 0.60 \\
\hline $0.1 \ldots \ldots \ldots$ & 3.99 & 96.01 & 3.99 & 1.04 & 0.62 \\
\hline $0.2 \ldots \ldots \ldots$ & 10.35 & 89.65 & 6.36 & 1.10 & 0.66 \\
\hline $0.3 \ldots \ldots$ & 17.46 & 82.54 & 7.11 & 1.18 & 0.71 \\
\hline $0.4 \ldots \ldots \ldots \ldots$ & 24.70 & 75.30 & 7.24 & 1.26 & 0.76 \\
\hline$\ldots \ldots$ & 31.77 & 68.23 & 7.07 & 1.34 & 0.81 \\
\hline $0.6 \ldots$ & 38.51 & 61.49 & 6.74 & 1.43 & 0.86 \\
\hline $0.7 \ldots \ldots$ & 44.81 & 55.19 & 6.30 & 1.52 & 0.91 \\
\hline $0.8 \ldots \ldots \ldots \ldots$ & 50.64 & 49.36 & 5.83 & 1.61 & 0.97 \\
\hline $0.9 \ldots \ldots \ldots \ldots$ & 55.97 & 44.03 & 5.33 & 1.70 & 1.02 \\
\hline $1.0 \ldots \ldots \ldots$ & 60.84 & 39.16 & 4.87 & 1.79 & 1.07 \\
\hline $1.1 \ldots \ldots \ldots \ldots$ & 65.24 & 34.76 & 4.40 & 1.89 & 1.13 \\
\hline $1.2 \ldots \ldots \ldots \ldots$ & 69.22 & 30.78 & 3.98 & 1.98 & 1.19 \\
\hline $1.3 \ldots \ldots \ldots \ldots$ & 72.75 & 27.25 & 3.53 & 2.08 & 1.25 \\
\hline $1.4 \ldots$ & 75.93 & 24.07 & 3.18 & 2.18 & 1.31 \\
\hline$\ldots \ldots$ & 78.77 & 21.23 & 2.84 & 2.27 & 1.36 \\
\hline $1.6 \ldots \ldots \ldots \ldots$ & 81.30 & 18.70 & 2.53 & 2.37 & 1.42 \\
\hline $1.7 \ldots \ldots \ldots \ldots$ & 83.54 & 16.46 & 2.24 & 2.46 & 1.48 \\
\hline $1.8 \ldots \ldots \ldots$ & 85.53 & 14.47 & 1.99 & 2.56 & 1.54 \\
\hline $1.9 \ldots \ldots \ldots$ & 87.28 & 12.72 & 1.75 & 2.66 & 1.60 \\
\hline 2.0 & 88.84 & 11.16 & 1.56 & 2.76 & 1.66 \\
\hline 2.2 . & 91.42 & 8.58 & 2.58 & 2.96 & 1.78 \\
\hline 2.4 & 93.48 & 6.52 & 2.06 & 3.18 & 1.91 \\
\hline 2.6 & 94.96 & 5.04 & 1.48 & 3.41 & 2.05 \\
\hline $2.8 \ldots \ldots \ldots \ldots$ & 96.16 & 3.84 & 1.20 & 3.63 & 2.18 \\
\hline $3.0 \ldots$ & 97.07 & 2.93 & .91 & 3.85 & 2.31 \\
\hline $3.5 \ldots \ldots \ldots \ldots$ & 98.52 & 1.48 & 1.45 & 4.44 & 2.66 \\
\hline 4.0. & 99.26 & .74 & .74 & 5.12 & 3.07 \\
\hline $5.0 \ldots$ & 99.72 & .28 & .46 & 6.15 & 3.69 \\
\hline $6.0 \ldots \ldots \ldots$ & 99.88 & .12 & .16 & 7.00 & 4.20 \\
\hline
\end{tabular}

Applying this to the curves that we have obtained, we get the information given in Tables V. and VI. Here we have assumed that at the 16.0- 
volt break we can express the magnitude of the new effect coming in as a function of the number of electrons with sufficient energy to produce the effect. If no new effect were coming in, the curve would continue to increase smoothly and, supposedly, would tend to flatten out, but the new effect starts the curve upward again. In the early part of the curve this effect is due only to a few of the electrons, but, with increasing voltage, more are able to produce the effect. It is assumed that in this interval of about a volt or a volt and a half the major part of the difference between the extrapolated curve and the observed curve is due to the distribution of velocities. To obtain a significant quantity the difference between the observed and extrapolated curves was divided by the percentage of electrons with total energy sufficient to take part in producing the effect. If we are correct in assuming that all electrons with energies greater than 16 volts are equally likely to produce ionization at impact (which is probably nearly true in the small range of velocities

TABLE V.

\begin{tabular}{|c|c|c|c|c|c|c|c|c|c|c|c|}
\hline \multirow{2}{*}{$\begin{array}{l}\text { Accel. } \\
\text { Poten., } \\
\text { Volts. }\end{array}$} & \multicolumn{2}{|c|}{ Correction. } & \multirow{2}{*}{$\begin{array}{l}\text { Per Cent. } \\
\text { having } \\
\text { this } \\
\text { Correc- } \\
\text { tion. }\end{array}$} & \multicolumn{4}{|c|}{ Grid off for Radiation. } & \multicolumn{4}{|c|}{ Grid on for Radiation. } \\
\hline & Volts. & $\vec{E}$. & & $\begin{array}{c}\text { Read- } \\
\text { ing. }\end{array}$ & $\begin{array}{c}\text { Extr. } \\
\text { Curve. }\end{array}$ & $\begin{array}{c}\text { Differ- } \\
\text { ence. }\end{array}$ & $\frac{\text { Diff. }}{\% .}$ & $\begin{array}{c}\text { Read- } \\
\text { ing. }\end{array}$ & $\begin{array}{l}\text { Extr. } \\
\text { Curve. }\end{array}$ & $\begin{array}{l}\text { Differ- } \\
\text { ence. }\end{array}$ & $\frac{\text { Diff. }}{\% .}$ \\
\hline 15.00 & 1.75 & 2.92 & 2.8 & 2.8 & 2.8 & 0.0 & 0.00 & 2.5 & 2.5 & 0 & .00 \\
\hline 15.25 & 1.50 & 2.50 & 4. & 3. & 3 & 0 & 0.02 & 3.1 & 2.9 & 0.2 & .05 \\
\hline 15.50 & 1.25 & 2.09 & 7. & 4.2 & 3. & 0.6 & 0.08 & 4.0 & 3.1 & 0.9 & .12 \\
\hline 15.75 & 1.00 & 1.67 & 12.7 & 5.8 & 4.0 & 1.8 & 0.14 & 5.1 & 3.4 & 1.7 & .13 \\
\hline 16.00 & 0.75 & 1.25 & 21.5 & 7. & 4. & 3.0 & 0.14 & 6.6 & 3.8 & 2.8 & .13 \\
\hline 16.25 & 0.50 & 0.84 & 37 & 9.2 & 4.8 & 4.4 & 0.12 & 8.6 & 4.1 & 4.5 & .12 \\
\hline 16.50 & 0.25 & 0.42 & 62 & 12.9 & 5.2 & 7.7 & 0.12 & 11.9 & 4.5 & 7.4 & .12 \\
\hline 16.75 & 0.00 & 0.00 & 100 & 16.1 & 5.6 & 10.5 & 0.11 & 14.5 & 4.8 & 9.7 & .10 \\
\hline
\end{tabular}

TABle VI.

\begin{tabular}{|c|c|c|c|c|c|c|c|c|c|c|c|c|}
\hline \multirow{2}{*}{$\begin{array}{l}\text { Accel. } \\
\text { Poten. } \\
\text { Volts. }\end{array}$} & \multicolumn{2}{|c|}{ Correction. } & \multirow{2}{*}{$\begin{array}{c}\text { Per } \\
\text { Cent. } \\
\text { Having } \\
\text { this } \\
\text { Correct. }\end{array}$} & \multirow{2}{*}{$(\%)^{3 / 2}$} & \multicolumn{4}{|c|}{ Grid off for Ionization. } & \multicolumn{4}{|c|}{ Grid on for Ionization. } \\
\hline & Volts. & $\vec{E}$ & & & $\begin{array}{c}\text { Read- } \\
\text { ing. }\end{array}$ & $\begin{array}{l}\text { Extr. } \\
\text { Curve. }\end{array}$ & $\begin{array}{l}\text { Differ- } \\
\text { ence. }\end{array}$ & $\frac{\text { Diff. }}{(\%)^{3 / 2}}$ & Read. & $\begin{array}{c}\text { Extr. } \\
\text { Curve. }\end{array}$ & $\begin{array}{c}\text { Differ- } \\
\text { ence. }\end{array}$ & $\frac{\text { Diff. }}{(\%)^{3 / 2}}$ \\
\hline 15.00 & 1.75 & 2.92 & 2.8 & 4.7 & 0.4 & 0.4 & 0.0 & 0.000 & 1.3 & 1.3 & 0.0 & 0.000 \\
\hline 15.25 & 1.50 & 2.50 & 4.5 & 9.6 & 0.5 & 0.4 & 0.1 & 0.010 & 1.6 & 1.4 & 0.2 & 0.022 \\
\hline 15.50 & 1.25 & 2.09 & 7.5 & 20.6 & 0.9 & 0.4 & 0.5 & 0.024 & 2.0 & 1.6 & 0.4 & 0.019 \\
\hline 15.75 & 1.00 & 1.67 & 12.7 & 45.6 & 1.8 & 0.4 & 1.4 & 0.031 & 2.8 & 1.8 & 1.0 & 0.022 \\
\hline 16.00 & 0.75 & 1.25 & 21.5 & 100 & 4.1 & 0.5 & 3.6 & 0.036 & 4.2 & 2.0 & 2.2 & 0.022 \\
\hline 16.25 & 0.50 & 0.84 & 37 & 227 & 8.7 & 0.5 & 8.2 & 0.036 & 7.1 & 2.1 & 5.0 & 0.022 \\
\hline 16.50 & 0.25 & 0.42 & 62 & 490 & 15.2 & 0.5 & 14.7 & 0.030 & - & - & - & - \\
\hline 16.75 & 0.00 & 0.00 & 100 & 1,000 & 23.2 & 0.6 & 22.6 & 0.023 & - & - & - & 一 \\
\hline
\end{tabular}


considered) then this ratio should be constant. For the radiation curves as given in Table $\mathrm{V}$. we see that the constancy of the ratio is well established from 15.75 to 16.50 , a region about one volt in length. This is about as much as can be expected. In these calculations, though provision is made for the increasing effect of the new break, we have not made the further correction in the falling off of the old break. If this is done Tables V. and VI. are changed to Table VII. In Table VI. we

TABLE VII.

\begin{tabular}{c|c|c|c|c}
\hline $\begin{array}{c}\text { Accel. Poten. } \\
\text { Volts. }\end{array}$ & $R$ (off) $\frac{\text { Diff. }}{\% .}$ & $R$ (on) $\begin{array}{c}\text { Diff. } \\
\% .\end{array}$ & $I$ (off) $\frac{D i f f .}{(\%)^{3 / 2} .}$ & $I$ (on) $\frac{\text { Diff. }}{(\%)^{3 / 2} .}$ \\
\hline 15.00 & 0.03 & 0.03 & 0.000 & 0.000 \\
15.25 & 0.07 & 0.07 & 0.010 & 0.031 \\
15.50 & 0.12 & 0.15 & 0.024 & 0.029 \\
15.75 & 0.18 & 0.17 & 0.033 & 0.026 \\
16.00 & 0.18 & 0.17 & 0.038 & 0.026 \\
16.25 & 0.17 & 0.16 & 0.037 & 0.026 \\
16.50 & 0.18 & 0.16 & 0.031 & \\
16.75 & 0.16 & 0.15 & 0.023 & \\
\hline
\end{tabular}

note that in order to obtain even an approximation to linearity the threehalves power of the per cent. must be used. This only goes to show that conditions in the tube are different in the two types of effect. It may well be that when a charged nucleus is accelerated only in the direction of emission of the electrons the effects due to it are linear but when it is forced to move in a curved path it obeys a different law. This is the case in the present problem. When the connections are arranged for observing radiation and ionization together, the charged molecules or atoms move on toward the plate in straight lines. On the other hand, in the arrangement to observe only ionization these particles are accelerated in a direction different from that in which they were moving. For this reason they will be traveling in orbits which will approximate parabolas with the wire point as their foci. Only in the case when the attraction is sufficiently great to bring the orbit within the small region occupied by the wire will the effect of the charged particle in the orbit be recorded. Another factor tending to increase the effect in the ionization curves is that more molecules and atoms may participate in collisions which will give observable effects. This is illustrated in Figs. Io and II. In Fig. Io, all the collisions must take place in the neighborhood of the gauze while in Fig. II, the collisions may take place in the whole region surrounded by the gauze and cup which are kept at the same potential. We find by a study of the results that the effect is increased so that it varies with the three-halves power of the accelerating voltage. 
Up to this point the question of the determination of the position of the break point has been based on an arbitrary assumption that the curve assumes a more or less linear character both before and after the break point. Nothing has been mentioned to indicate whether this type

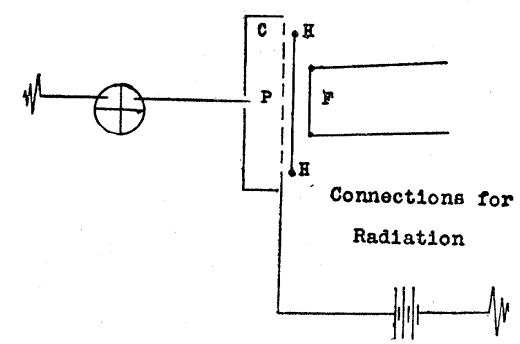

Fig. 10.

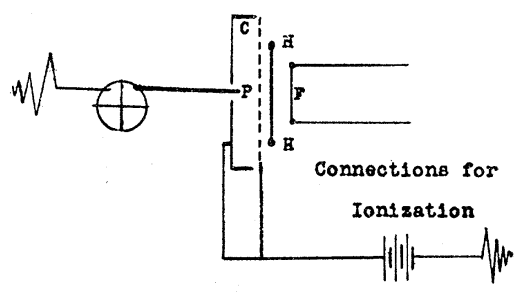

Fig. 11.

of determination of the break should be more or less accurate than the usual one of taking the point of rise in the curve as the real break point. It has just been shown, however, that the curve has a roundness at the break point providing the break is so pronounced that the effect of the distribution of velocities becomes an important factor. If we consider the weaker breaks, which do not have this large distribution of velocity factor, we find that the curve on the two sides of the break is almost given by straight lines which are inclined to each other at a small angle. This means that if we were able to obtain a more accurate set of values near this point and could plot them on a larger scale we would find a similar distribution of velocity curve. Conversely, if we should plot on a much smaller scale the curve near the stronger breaks, where we observe the distribution of velocity correction, we would find that the limit of the curve would be two straight lines intersecting at the break point. This is the real significance of this criterion for a break. It actually makes the position of the break independent of whether the effect observed is large or small. Now concerning the accuracy with which the break is fixed, we must again consider these two types of effect. The large effects are sometimes so large that the scale must change at least once in passing through the break point. This means that we must use care in selecting the particular curve which gives the correct break point. Obviously the only one to use is the one to a scale which can show the curve for at least one or two volts on either side of the break point. The intersection of the tangents which most nearly express the remaining portion of the curve on either side of the break then gives the correct break point. We find that this gives a probable error of about .2 volt in almost every case. Thus we obtain all breaks properly spaced 
relatively to each other, independent of the velocity distribution, if we determine the position of each when plotted to a scale giving a difference of slope on each side of the break which is the same for all.

We must also try to determine the absolute accuracies of the points of discontinuity of each curve. Turning to the spectroscopic evidence we note that the IO.I-volt and the I3.5-volt breaks can be accurately computed while the breaks which are due to the molecule do not have this additional check. We have already shown that the accuracy of the big break is \pm .2 volt, so we can easily compare the points at which the breaks occur and then discuss the accuracy of the positions. If we compare the values for the small breaks with the values predicted on the Bohr Theory and find how they are fixed relative to each other we find that the agreement is extremely good. So we are left with only one break to fix and we have one bit of evidence that helps us to fix that relatively to the others. Among the small breaks is the one for "dissociation of the molecule plus radiation from an atom." This was found to be 2.8 volts higher than "radiation from the atom." This 2.8 volts is therefore the energy required for dissociation of the molecule. That means that in order to fix the value for "dissociation of the molecule plus ionization from one atom" we must have from the figures which are given $\mathrm{I} 3.6+2.8$ or $\mathrm{I} 6.4$ volts. The result obtained was $\mathrm{I} 6.0 \pm .2$ volts so that the values check with a maximum accuracy of .2 volt, or to a minimum accuracy of .6 volt. The most probable tendency is to place the big break too soon, for the observer is always anxious to note the beginning of an effect though it might be better for him to determine the point at which the full effect comes in rather than any intermediate point. In this case we might suppose that the small breaks were observed only when the velocity of all electrons was sufficient to give the observed effect. This would correspond, in the case of the big break, to the point at which the intermediate curve had a slope corresponding to the slope of the curve beyond the break. This adds about .5 volt to the previous observed value, giving us 16.5 as a better position of the break. This seems to agree a bit better with the other calculation. However, these suggestions are such as to show that the results are in good agreement and are really not sufficient to definitely settle the exact position of the big break. Probably this last correction, which brings the value up to I6.5 volts, is in reality too much and the actual position is somewhere between 16.0 and I6.5 volts. This seems the only logical conclusion at which we can safely arrive. 


\section{Conclusion.}

As a result of this research, Bohr's Theory of the Hydrogen Atom has been experimentally closely verified, since both the first and second radiation potentials, corresponding to the first two terms of the Lyman Series, have been obtained. In addition it has been conclusively shown that two ionizing potentials of the molecule exist, the first at I I.5 volts leaving the molecule positively charged, while the second at I6.0-I6.5 volts dissociates the molecule and ionizes one of its parts.

In conclusion, the writer wishes to express his sincere thanks to Professor K. T. Compton for his suggestions and assistance in carrying out the investigation.

PALMer Physical Laboratory,

Princeton University,

June, r922.

Note: Fig. Io should show the point $P$ and the cup $C$ electrically connected and insulated from the gauze. 